\title{
Hepatoma-derived growth factor stimulates smooth muscle cell growth and is expressed in vascular development
}

\author{
Allen D. Everett, ${ }^{1,2}$ David R. Lobe, ${ }^{3}$ Martin E. Matsumura, ${ }^{3}$ Hideji Nakamura, ${ }^{4}$ \\ and Coleen A. McNamara ${ }^{2,3}$ \\ ${ }^{1}$ Department of Pediatrics, \\ ${ }^{2}$ Cardiovascular Research Center, and \\ ${ }^{3}$ Department of Internal Medicine, Cardiovascular Division, University of Virginia Health Sciences Center, \\ Charlottesville, Virginia 22908, USA \\ ${ }^{4}$ Department of Medicine III, Osaka University Medical School, Osaka 618, Japan \\ Address correspondence to: Allen D. Everett, University of Virginia, MR4 Building, Box 14, Charlottesville, \\ Virginia 22908, USA. Phone: (804) 982-0260; Fax: (804) 982-4387; E-mail: ade5r@virginia.edu.
}

Received for publication June 3, 1999, and accepted in revised form January 26, 2000.

Hepatoma-derived growth factor (HDGF) is the first member identified of a new family of secreted heparin-binding growth factors highly expressed in the fetal aorta. The biologic role of HDGF in vascular growth is unknown. Here, we demonstrate that HDGF mRNA is expressed in smooth muscle cells (SMCs), most prominently in proliferating SMCs, 8-24 hours after serum stimulation. Exogenous HDGF and endogenous overexpression of HDGF stimulated a significant increase in SMC number and DNA synthesis. Rat aortic SMCs transfected with a hemagglutinin-epitope-tagged rat HDGF cDNA contain HA-HDGF in their nuclei during S-phase. We also detected native HDGF in nuclei of cultured SMCs, of SMCs and endothelial cells from 19-day fetal (but not in the adult) rat aorta, of SMCs proximal to abdominal aortic constriction in adult rats, and of SMCs in the neointima formed after endothelial denudation of the rat common carotid artery. Moreover, HDGF colocalizes with the proliferating cell nuclear antigen (PCNA) in SMCs in human atherosclerotic carotid arteries, suggesting that HDGF helps regulate SMC growth during development and in response to vascular injury.

J. Clin. Invest. 105:567-575 (2000).

\section{Introduction}

Smooth muscle cell (SMC) proliferation is a key component of vascular development and vascular injury (1-4). Many extracellular growth factors liberated at the time of vascular injury are known to stimulate SMC proliferation in vitro (5-7). Initial studies focused interest on growth factors released from blood-borne cells such as platelets (7). Vascular injury induces platelet aggregation with resultant local release of PDGF, a potent SMC mitogen. However, subsequent studies have shown that intimal SMC proliferation occurred with no platelets in the vascular lumen $(8,9)$, suggesting that vascular cells may be the source of their own mitogens. Indeed, PDGF is produced by many cell types in vivo and in vitro, including vascular SMCs and endothelial cells $(7,10)$. Vascular wall cells also synthesize other SMC mitogens including the heparin-binding growth factors such as FGF (11). The mechanisms responsible for the expression of growth factors in vascular cells and their role in control of growth and differentiation in SMCs after vascular injury are poorly understood. Clearly, a fuller understanding of the SMC response to vascular injury will involve identification of all the factors involved in SMC growth and differentiation in the vessel wall during development and in response to injury.

Hepatoma-derived growth factor (HDGF) is a heparin-binding growth factor originally purified from the human hepatoma-derived cell line, HuH-7 (12).
HDGF is a secreted growth factor; however, its sequence contains a putative nuclear localization cassette. HDGF is ubiquitously expressed and is mitogenic for fibroblasts, HuH-7 cells (13), and aortic endothelial cells (14). Although the specific function of this protein is unknown, recent studies have suggested a role for HDGF in the regulation of renal vascular development (14). We recently identified and cloned the rat homologue of HDGF as a developmentally regulated mRNA in the developing rat embryonic aortic sac (9).

Results of the present study demonstrated that HDGF mRNA was expressed in cultured vascular SMCs. HDGF protein was detected in the nucleus of cultured SMCs, and exogenous HDGF and endogenous overexpression of HDGF stimulated SMC proliferation. HDGF protein was also detected in the nucleus of rat fetal, but not adult, aortic SMCs, but was re-expressed in the nucleus of SMCs in the adult rat aorta after aortic banding. HDGF protein was detected in the adult rat carotid after balloon endothelial denudation and in regions of human carotid atherosclerotic plaques that contain proliferating SMCs. Taken together, these results suggest that HDGF is a growth factor that is involved in stimulating SMC proliferation during development and in disease.

\section{Methods}

Cell culture. Rat aortic SMCs (a generous gift from G. Owens, University of Virginia) were grown in 


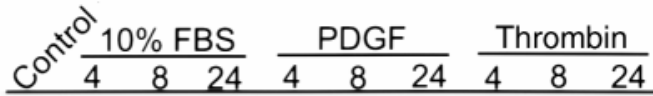

HDGF

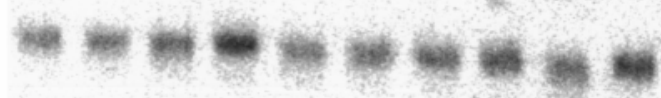

GAPDH

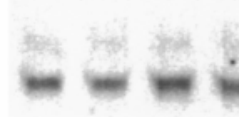

\section{Figure 1}

Northern blot of HDGF mRNA expression in subconfluent vascular SMCs in response to serum, PDGF, or thrombin. SMCs were quiesced in SFM for 48 hours and treated with 10\% FBS, PDGF (10 $\mathrm{ng} / \mathrm{mL})$, or thrombin $(100 \mathrm{nM})$ for 4-24 hours. Total RNA (10 $\mu \mathrm{g})$ was isolated and analyzed for HDGF mRNA expression, and GAPDH was used as a control for loading.

DMEM:F12 (1:1) with 10\% FBS, 10 U penicillin, and 10 $\mu \mathrm{g}$ streptomycin per milliliter (GIBCO BRL, Grand Island, New York, USA). To induce quiescence, the cells are placed in a defined serum-free medium (SFM) containing DMEM:F12 (1:1) with $10 \mathrm{U}$ penicillin and 10 $\mu \mathrm{g}$ streptomycin per milliliter, $35 \mu \mathrm{g} / \mathrm{mL}$ ascorbic acid, $5 \mu \mathrm{g} / \mathrm{mL}$ transferrin, $2.85 \mu \mathrm{g} / \mathrm{mL}$ insulin, and 6.25 $\mathrm{ng} / \mathrm{mL}$ selenium (all from Sigma Chemical Co., St. Louis, Missouri, USA) for 48 hours.

$R N A$ analysis. SMCs were lysed in Tri-Reagent (Molecular Research Center, Cincinnati, Ohio, USA), and total RNA was recovered. Ten micrograms of total RNA was electrophoresed under denaturing conditions in a $1.2 \%$ agarose/MOPS/formaldehyde gel and transferred to a charged nylon membrane as described previously (15). A 1,620-bp full-length rat HDGF cDNA determined by bidirectional sequencing (includes the 720 bp open reading frame and $900 \mathrm{bp}$ of $3^{\prime}$ noncoding sequence) was recovered from screening a 14-day fetal rat heart cDNA library with a 300-bp PCR fragment recovered from differential display of 12-day fetal rat heart outflow tract and truncus. The 1,620-bp rat HDGF cDNA was radiolabeled by random priming, hybridized overnight at $65^{\circ} \mathrm{C}$, and washed (15). The blots were also probed with the cDNA for GAPDH as a control for loading and gene specific expression (15). Specific HDGF mRNA hybridization was detected using a phosphorimager (Molecular Dynamics, Sunnyvale, California, USA).

Transfection studies. The 1,620-bp rat HDGF cDNA was subcloned into mammalian expression vectors to produce an amino hemagluttinin (pKH3) or GFP (pK7GFP) epitope tag HDGF fusion (a generous gift of I. Macara, University of Virginia). Cultured rat aortic SMCs were plated onto coverslips in $60-\mathrm{mm}$ plates at a density of $2 \times 10^{4} / \mathrm{cm}^{2}$. Twenty-four hours after plating, cells were transfected with $5 \mu \mathrm{g}$ of control vector $\mathrm{pKH} 3$ or pKH3-HDGF using $30 \mu \mathrm{g}$ of DOTAP transfection reagent (Boehringer-Mannheim, Indianapolis, Indiana, USA) per manufacturer's protocol. Cultures were main- tained in growth media (DF10), pulsed with BrdU (Boehringer-Mannheim) $8(n=3)$ and $36(n=3)$ hours after transfection, and fixed for immunostaining at 24 and 48 hours after transfection.

For proliferation studies, SMCs were also transfected with $\mathrm{pKH} 3$ or $\mathrm{pKH} 3-\mathrm{HDGF}$ and AdLox-GFP as a transfection control. Transfected SMCs were fluorescent cell sorted for GFP-expressing cells and replated at a density of 1,000 cells per well in a 96-well plate in DF10 media. Cells were assayed for cell number (Celltiter; Promega Corp., Madison, Wisconsin, USA) at 24, 48, and 72 hours. Cell number was determined from a standard curve of 1,000-60,000 cells per well.

Western analysis. Human colon carcinoma cells were transfected with pKH3-HDGF cDNA and cultured for 48 hours. The media was aspirated, and the cells were washed 2 times with cold Hanks' buffered saline (GIBCO BRL) and scraped in TSA lysis buffer (TSA:0.002 M Tris Cl [pH 8.0], 0.14 M NaCl, 0.025\% $\mathrm{NaN}_{3}, 1 \%$ Tween- $20,0.5 \%$ sodium deoxycholate $+1 \mathrm{mM}$ PMSF, aprotinin $2 \mathrm{U} / \mathrm{mL}$ ). The cells were lysed by 3 cycles of freeze/thawing, and soluble proteins were recovered after centrifugation at $10,000 \mathrm{~g}$ for 30 minutes at $4^{\circ} \mathrm{C}$. For immunoprecipitation, $100 \mu \mathrm{L}$ of cell lysate

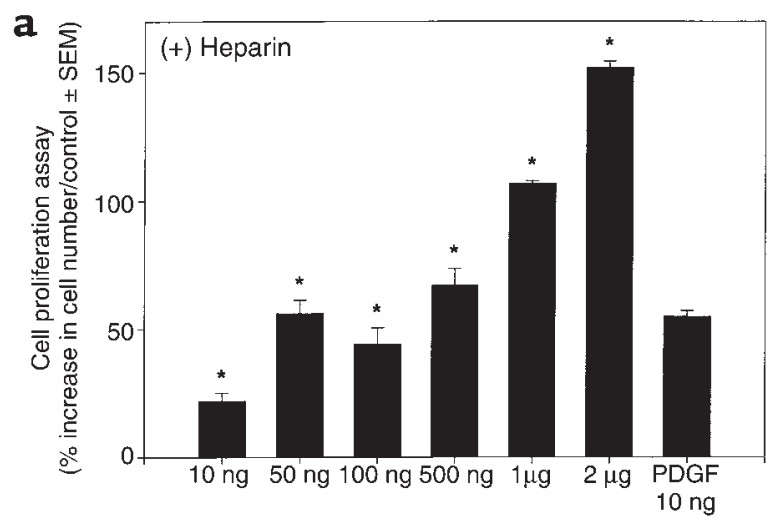

b

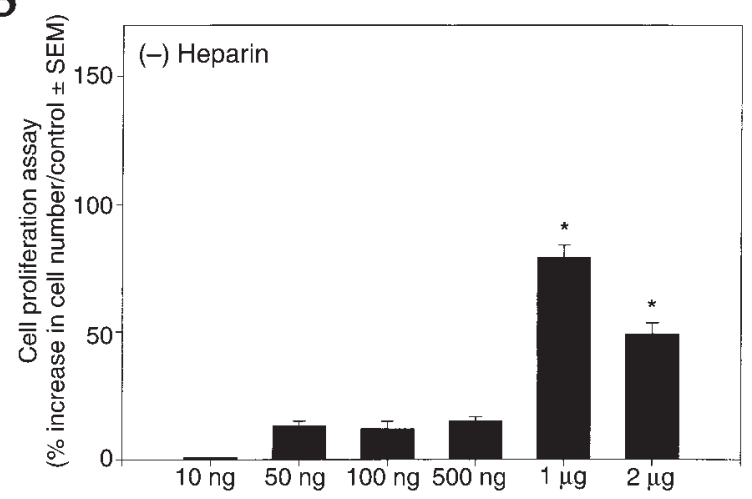

\section{Figure 2}

Effect of exogenous recombinant HDGF on SMC proliferation. SMCs in SFM with (a) or without (b) heparin were cultured for 72 hours with vehicle (control) or HDGF $(10 \mathrm{ng}-2 \mu \mathrm{g} / \mathrm{mL})$ and assayed for cell number. Cell numbers per condition were expressed as percent increase over controls. For controls, $n=8$; for HDGF, $n=4$ per concentration. The entire experiment was repeated twice in 2 separate experiments. ${ }^{*} P<0.05$ by one-way ANOVA control versus HDGF. 


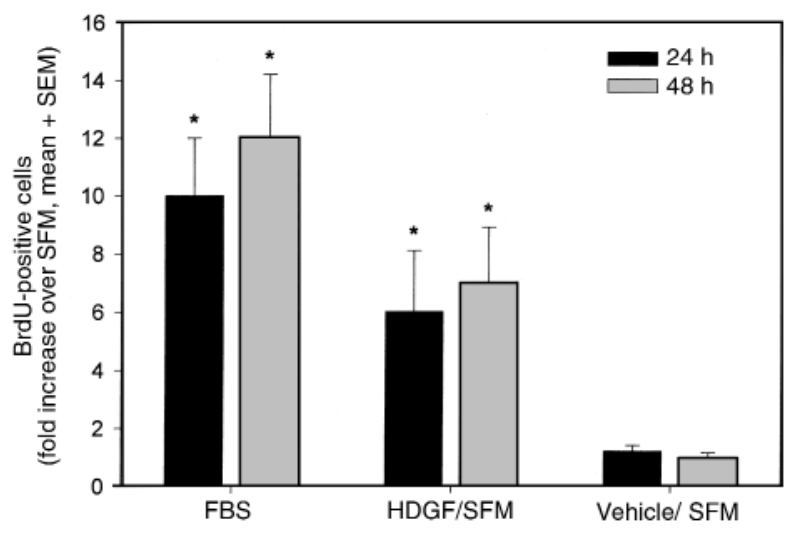

Figure 3

Effect of exogenous HDGF on BrdU uptake in SMCs. SMCs grown on coverslips in SFM were treated with SFM, 10\% FBS, HDGF (100 $\mathrm{ng} / \mathrm{mL}$ ) or vehicle (PBS) for 24 or 48 hours and pulsed with BrdU for 6 hours. BrdU-positive nuclei were counted ( $n=4$ coverslips per group) and expressed as fold increase over SFM group as mean \pm SEM. ${ }^{*} P<0.05$ SFM versus FBS or HDGF by one-way ANOVA.

was diluted to $500 \mu \mathrm{L}$ with lysis buffer and incubated with $1 \mu \mathrm{g}$ of an anti-hemagglutinin (anti-HA) $\mathrm{mAb}$ (Santa Cruz Biotechnology Inc., Santa Cruz, California, USA) bound to protein A Sepharose (Sigma Chemical Co.) for 1 hour at $4^{\circ} \mathrm{C}$. The immunoprecipitated HAHDGF was subjected to immunoblotting with a $\mathrm{COOH}$ terminal affinity-purified HDGF antibody detected by enhanced chemiluminescence (ECL) autoradiography (Amersham Pharmacia, Piscataway, New Jersey, USA).

Nuclear extracts were prepared from rat aortic SMCs grown in $225-\mathrm{cm}^{2}$ flasks in DMEM-F12. The cells were harvested, and nuclear extracts were prepared by the procedure of Dignam et al. (16). A total of $50 \mu \mathrm{g}$ of nuclear protein was resolved by $10 \%$ SDS-PAGE and blotted with the HDGF antibody and detection by ECL autoradiography.

Recombinant HDGF. Recombinant HDGF was produced as a GST-HDGF fusion protein. The vector pGEX-3X (Amersham Pharmacia) was digested with BamHI and end-filled by T4 DNA polymerase and was subsequently digested by EcoRI. An NruI and EcoRI fragment of the HDGF cDNA (12), containing the entire open reading frame, was then cloned into pGEX$3 \mathrm{X}$. This produced an $\mathrm{NH}_{2}$-terminus of recombinant HDGF as G-I-R-S-N- instead of S-R-S-N- of native HDGF. The lysate of Escherichia coli was incubated with glutathione-sepharose beads at $4^{\circ} \mathrm{C}$, washed thoroughly with PBS and recombinant HDGF released by Factor $\mathrm{Xa}$, and purified on a heparin-sepharose column.

Proliferation assays. SMCs were plated onto 96-well plates at a density of $3 \times 10^{3}$ cells per well. Cells were grown to confluence, changed to SFM for 5 days, and then stimulated with $10 \mu \mathrm{L}$ of HDGF vehicle as a control or recombinant HDGF $(10 \mathrm{ng}-2 \mu \mathrm{g} / \mathrm{mL})$. At 72 hours after stimulation, cell number was measured using a colorimetric cell proliferation assay (Celltiter Aqueous 1 reagent; Promega Corp.).
$B r d U$ and TUNEL assays. BrdU and TUNEL studies were performed with SMCs grown on plastic coverslips $(n=3$ coverslips per group). For BrdU studies, SMCs quiesced in SFM for 48 hours were treated with SFM alone, vehicle (saline), HDGF (100 ng/mL), or 10\% FBS and pulsed with $\operatorname{BrdU}(1 \mu \mathrm{M})$ for 6 hours before harvesting at 24 and 48 hours after treatment. Cells positive for BrdU incorporation were determined by immunocytochemistry with an alkaline phosphatase conjugated anti-BrdU antibody (Boehringer-Mannheim) and counted. The number of BrdU-positive cells in each group were compared with the SFM alone group.

For the TUNEL assay, SMCs were treated with SFM alone, SFM plus vehicle, HDGF $(100 \mathrm{ng} / \mathrm{mL})$ in SFM, or $10 \mathrm{FBS} \%$. At 24 and 48 hours, cells were fixed in $1 \%$ paraformaldehyde and TUNEL labeling was performed using a commercial kit (Boehringer-Mannheim) and the Vector Red substrate. TUNEL-positive nuclei were counted, and the fold increase in TUNEL-positive nuclei was compared with the FBS group.

Immunostaining. Transfected SMCs on coverslips were fixed at $-20^{\circ}$ in $70 \%$ ethanol and allowed to air dry. Coverslips were blocked for 1 hour in 3\% BSA/PBS (Boehringer-Mannheim) and incubated with an antiBrdU alkaline phosphatase conjugated antibody (1:500; Boehringer-Mannheim) and a monoclonal anti-HA-epitope tag antibody (1:200; Santa Cruz Biotechnology) for 1 hour at room temperature. An FITC-conjugated antimouse secondary antibody (1:200; Amersham Pharmacia) and a red alkaline phosphatase substrate (Vector Laboratories, Burlingame, California, USA) were used for viewing. The coverslips were subsequently rinsed with tap water and inverted onto microscope slides containing a drop of mounting media (Vectashield; Vector Laboratories) for visual inspection with an Olympus BX5OWI Fluoview confocal microscope.

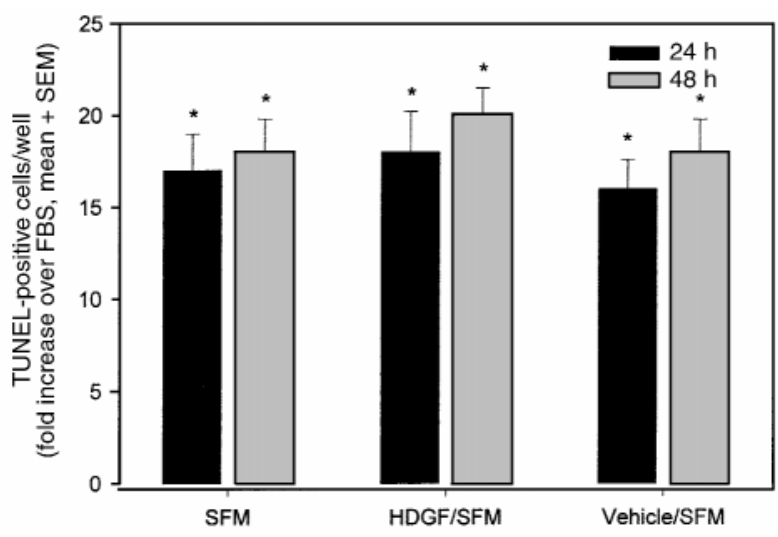

\section{Figure 4}

Effect of exogenous HDGF on the induction of apoptosis. SMC grown on coverslips were cultured in SFM alone, SFM + HDGF (100 $\mathrm{ng} / \mathrm{mL}), \mathrm{SFM}+$ vehicle (PBS), or $10 \%$ FBS for 24 or 48 hours. The TUNEL reaction was performed ( $n=4$ coverslips per group) and TUNEL-positive nuclei counted and expressed as the fold increase over the FBS group as mean \pm SEM. ${ }^{*} P<0.05$ versus the FBS group by one-way ANOVA. 


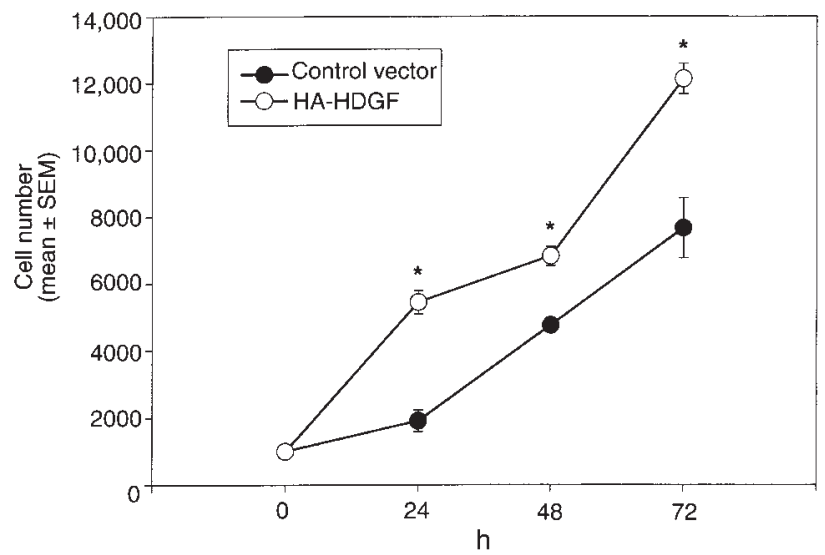

Figure 5

Effect of endogenous overexpression of HDGF on SMC proliferation. SMCs were transfected with $\mathrm{PKH} 3-\mathrm{HDGF}$ and the transfection control plasmid pAdLox-GFP or the empty vector pKH3 and pAdLoxGFP. Forty-eight hours after transfection, cells were fluorescent cell sorted and replated at 1,000 cells per well. Cell proliferation was assayed by ELISA at 24,48 , and 72 hours after plating ( $n=4$ per group, repeated 3 times). Cell numbers are expressed as mean \pm SEM. ${ }^{*} P<0.05$ control versus HDGF-transfected groups.

Tissue sections for HDGF immunostaining were blocked with normal goat serum (Vector Laboratories) for 1 hour, washed, and incubated with either an HDGF affinity-purified polyclonal antibody produced against the COOH-terminal amino acids 222-237 of the mouse HDGF sequence (17) at a dilution of 1:1000, FITC conjugated anti-smooth muscle $\alpha$-actin (1:500; Sigma), or FITC conjugated anti-PCNA (1:100; DAKO Corp., Carpinteria, California, USA). After washing with PBS/0.5\% Tween-20, HDGF immunodetection was performed using the ABC method (Vector Laboratories) with either DAB or Vector Red as a chromogenic substrate. Using this technique, HDGF-immunopositive cells were brown (DAB) or red (Vector Red). Controls for HDGF immunostaining included staining without the primary HDGF antibody, staining with nonimmune rabbit serum, and preabsorption of the primary HDGF antibody with the synthetic target peptide. All controls demonstrated no background immunostaining.

Tissue preparation

Rat carotid injury model. Male 300-g Sprague-Dawley rats (Harlan Bioproducts for Science Inc., Indianapolis, Indiana, USA) underwent carotid artery balloon injury as described previously (18). After recovery, a midline sternotomy was performed and the animals were pressure perfused with $4 \%$ paraformaldehyde in cold PBS. The injured left and control right carotids were removed and postfixed in 4\% paraformaldehyde for 2 hours, dehydrated in a graded alcohol-xylene series, and paraffin embedded for thin sectioning.

Human carotid endarterectomy specimens. Freshly excised human carotid plaques were collected at the time of carotid endarterectomy according to institution human tissue usage protocols. The samples were fixed for 2 hours in 4\% paraformaldehyde, dehydrated in a graded alcohol-xylene series, and mounted in paraffin for thin sectioning.

Fetal tissue harvest. Fetal (19-day gestation) thorax block dissections and aortas from 90-day-old adult rat thoracic aortas were removed and immersion fixed in ice cold $4 \%$ paraformaldehyde for 1 hour, embedded in OCT compound (Miles Inc., Elkart, Indiana, USA), and frozen by immersion in liquid nitrogen. Sections (4-7 $\mu \mathrm{m})$ were cut and thaw mounted onto glass slides.

Abdominal aortic constriction. Abdominal aortic constriction was performed as described previously (19). Adult Sprague-Dawley rats had constriction of the suprarenal abdominal aorta by a ligature. After 72 hours, the degree of aortic constriction was determined by simultaneous carotid and femoral artery blood pressure measurement using indwelling catheters (19). This technique routinely produced an average peak $50 \mathrm{mmHg}$ gradient across the area of aortic constriction. After blood pressure determinations, the aorta was perfusion fixed via the carotid artery catheter with $4 \%$ paraformaldehyde. The thoracic and abdominal aorta were subsequently dissected free, dehydrated in a graded alcohol-xylene series, and mounted in paraffin for thin sectioning.

\section{Results}

HDGF $m R N A$ is expressed in vascular SMCs. Vascular wall cells synthesize mitogens that can act in an autocrine or paracrine manner to regulate cell growth in development and in response to injury states. To determine whether HDGF is expressed in SMCs and in response to the mitogens PDGF and thrombin, Northern analysis of cultured rat aortic SMCs was performed. As shown in Figure 1, HDGF mRNA is expressed at baseline in SMC. When controlled for GAPDH, serum stimulation results in a 2.5 -fold increase in HDGF mRNA expression, peaking at 8-24 hours. However, PDGF or thrombin had no effect on HDGF mRNA levels.

HDGF stimulates SMC proliferation. HDGF is a known mitogen for endothelial cells in culture (14) and is expressed in cultured vascular cells (Figure 1). Whether HDGF plays a role in regulating SMC growth is unknown. To determine whether HDGF was an SMC mitogen, quiescent SMC were treated with HDGF vehicle or HDGF $(10 \mathrm{ng}-2 \mu \mathrm{g} / \mathrm{mL})$ with or without heparin. Cell number was measured 72 hours after stimulation. As shown in Figure 2a, HDGF stimulated a significant dose-dependent increase in cell number compared with control. At the lowest concentration used (10 ng), HDGF produced significant SMC proliferation $(22 \%$; $P<0.05)$. The necessity of heparin as a cofactor is demonstrated in Figure 2b, which shows that omission of heparin significantly blunts the HDGF proliferative response. The increase in cell number with HDGF treatment was due to increased DNA synthesis, as BrdU incorporation was increased with HDGF treatment (Figure 3) and not secondary to an antiapoptotic effect (Figure 4). 

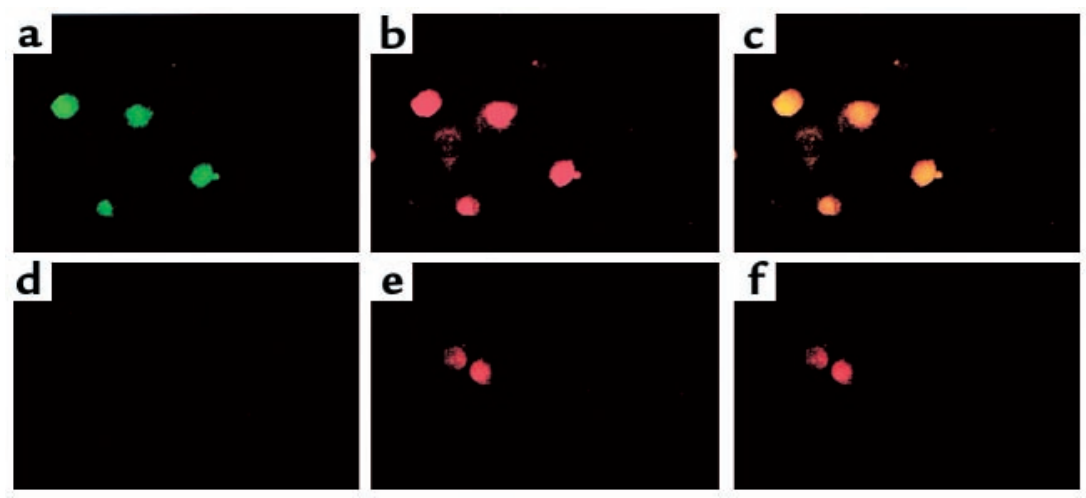

\section{Figure 6}

Effect of HDGF overexpression in SMCs on BrdU uptake and cell localization. SMCs were transfected with HA-epitope-tagged HDGF (a-c) or empty vector $(\mathbf{d}-\mathbf{f})$ and pulsed with BrdU as a marker of DNA synthesis for 12 hours. Cells were stained with an HA mAb and an FITCconjugated secondary antibody to detect HDGF-transfected cells (green, $\mathbf{a}$ and $\mathbf{d}$ ) and an alkaline phosphatase-conjugated anti-BrdU antibody to detect cells replicating their DNA (red, $\mathbf{b}$ and $\mathbf{e}$ ). $\mathbf{c}$ is an overlay of $\mathbf{a}$ and $\mathbf{b}$ demonstrating colocalization (yellow) of HDGF to the nucleus of cells replicating their DNA. $\mathbf{f}$ is an overlay of $\mathbf{d}$ and $\mathbf{e}$.

To examine whether endogenous cellular overexpression of HDGF increases SMC number, SMCs were cotransfected with the expression plasmids encoding green fluorescent protein (pAdLox-GFP) and an expression plasmid encoding a hemagglutinin-epitope tag (pKH3) with or without a full-length rat HDGF cDNA (pKH3-HDGF). Forty-eight hours after transfection, SMCs were fluorescent cell sorted to enrich for transfected cells and replated. As shown in Figure 5, when compared with pKH3-transfected cells, pKH3HDGF-transfected cells demonstrated a significant increase in their cell numbers at 24,48 , and 72 hours $(P$ $=0.05)$. Therefore cellular overexpression of HDGF enhances SMC number.

HDGF is a nuclear protein. HDGF mRNA is expressed in cultured vascular cells, demonstrating increased levels in cells that are proliferating (Figure 1). Additionally, application of exogenous and cellular overexpression of HDGF induced SMC proliferation in culture (Figures 2, 3 , and 5), suggesting that HDGF is a secreted protein that stimulates SMC growth through an autocrine or paracrine mechanism. However, the HDGF protein contains a putative nuclear localization signal. To determine whether HDGF traffics to the nucleus, cultured SMC cells were transfected with pKH3-HDGF, enabling detection of expressed HDGF via the HA-epitope tag, and pulsed with the thymidine analogue BrdU as a marker of DNA synthesis. As shown in Figure 6a (green cells), transfected SMCs express HA-HDGF in the nucleus as HA-HDGF and BrdU (red cells; Figure 6b) colocalize (Figure $6 \mathrm{~b}$ and in the overlay, c). Interestingly, every cell expressing HA-HDGF was also BrdU-positive, whereas adjacent cells not expressing HA-HDGF did not incorporate BrdU (Figure 6, a and b). Control cells transfected with the empty vector were negative for HA (Figure 6d) and were only BrdU-positive (Figure 6e). Targeting of HDGF to the nucleus was not an artifact of immunostaining, as expression of HDGF as an amino-GFP fusion in transfected SMCs was also targeted to the nucleus in living cells (Figure 7).

To determine whether native HDGF is normally expressed in the nucleus of cultured SMCs, nuclear protein extracts were prepared from cultured SMCs and assayed by Western analysis and immunoblotting with a COOH-terminal HDGF polyclonal antibody. To confirm that the HDGF antibody detected HDGF, human colon adenocarcinoma cells were transfected with HA-HDGF, immunoprecipitated with a monoclonal HA antibody, and immunoblotted with the HDGF antibody. As shown in Figure 8, the HDGF antibody identified a single $38-\mathrm{kDa}$ protein consistent with HDGF in cells transfected with the HA-HDGF $(+)$ construct and not with the vector alone (-). Endogenous HDGF was detected in SMC nuclear extracts as a predicted 38-kDa protein using the anti-HDGF antibody. Although HDGF was discovered as a secreted protein
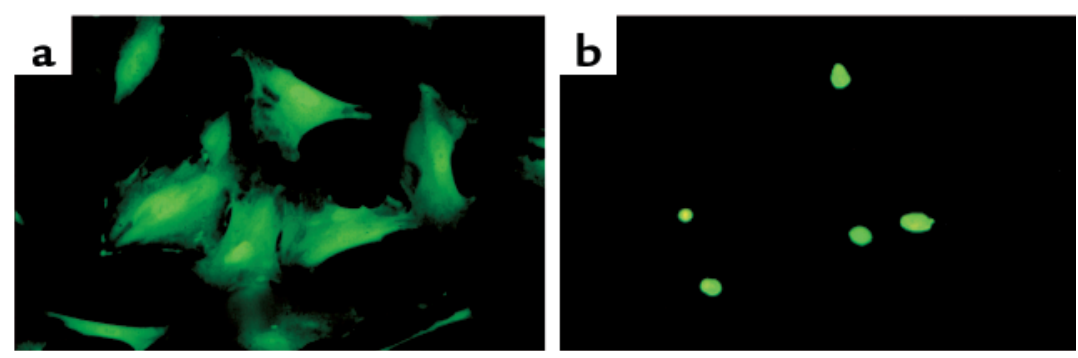

\footnotetext{
Figure 7

HDGF is targeted to the nucleus in living SMCs. SMCs were transfected with the empty vector (a, pK7-GFP) or (b) pK7-GFP-HDGF to express HDGF as an amino GFP fusion. HDGF expression was examined at 18 hours after transfection by fluorescent microscopy.
} 


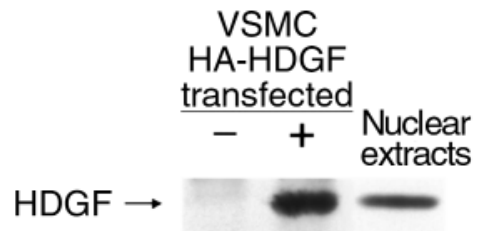

\section{Figure 8}

Native HDGF protein is located in the nucleus of cultured SMCs by Western analysis. Human adenocarcinoma cells were transfected with pKH3-HDGF $(+)$ or empty vector alone $(-)$. In lanes 1 and 2, recombinant $\mathrm{HA}$-epitope-tagged HDGF was immunoprecipitated with an $\mathrm{HA} \mathrm{mAb}$ from cellular extracts of transfected cells, and in lane 3 nuclear extracts from cultured SMCs were separated by $10 \%$ SDS-PAGE and blotted with an anti-HDGF polyclonal antibody.

in conditioned media, HDGF is also a nuclear protein in cultured SMC trafficking to the nucleus in SMCs, replicating their DNA.

HDGF is developmentally regulated in the rat aorta. To determine whether HDGF is present in vascular cells in vivo, immunocytochemistry with the anti-HDGF antibody was performed on 19-day fetal and adult rat aorta tissue sections. As shown in Figure 9a, HDGF is ubiquitously expressed in the nuclei of SMCs and endothelial cells in the fetal aorta. In contrast, detection of HDGF protein is dramatically decreased in the mature 90-dayold rat aorta, with only an occasional faintly positive cell present in the outer media/adventitia (Figure 9b).

HDGF expression is increased in rat aortic SMCs proximal to an aortic constriction. As HDGF expression is barely detectable in the mature aorta, we studied the effect of abdominal aortic constriction, a model known to produce both SMC proliferation and hypertrophy, on the modulation of HDGF expression in the aorta. As shown in Figure 10, few HDGF immunopositive cells are present in the thoracic (Figure 10a, arrows) or abdominal (Figure 10b) aorta in a representative sham-operated control animal. The HDGF-immunopositive cells were generally restricted to the outer layers of the media, or an occasional adventitial cell (Figure 10, a and b, arrows). The response of the aorta above and below abdominal aortic constriction is illustrated in Figure 10, $\mathrm{c}$ and $\mathrm{d}$. Aortic constriction produced an increase in the number of HDGF-positive nuclei in the media and adventitia of the thoracic aorta above the constriction (Figure 10c, brown nuclei), with no change in the number of HDGF positive nuclei in the abdominal aorta below the aortic constriction (Figure 10d, arrow).

HDGF is expressed in SMCs during neointima formation after balloon injury. The rat carotid injury model is a well-studied model of smooth muscle proliferation after endothelial injury (18). To determine whether HDGF is expressed in the proliferating SMCs in the neointima developed after balloon endothelial denudation, immunohistochemistry was performed.

As shown in Figure 11a, HDGF was not detected in the media of uninjured rat carotid arteries. Fourteen days after balloon injury, a large neointima develops (Figure 11b) as a result of SMC migration and proliferation (18). At this stage of injury, proliferative SMCs are restricted to the forming neointima. As shown in Figure 11b, HDGF was abundantly detected in proliferative neointimal, but not in the relatively nonproliferative medial, cells at this stage of injury. Twenty-eight days after balloon injury (Figure 11c), when the proliferative index has declined to near baseline levels (18), there is a marked decrease in the number of HDGFpositive cells (Figure 11c, arrows). The control section shown in Figure 11d, lacking the primary antibody, demonstrates the specificity of the staining.

HDGF is coexpressed with PCNA in SMCs of buman atherosclerotic carotid arteries. To determine whether HDGF was expressed in proliferating SMCs within human atherosclerotic lesions, immunohistochemistry was performed on human carotid endarterectomy specimens. These specimens contained large areas of plaque with calcified segments and are largely hypoproliferative; however, several discrete areas of SMC proliferation were detected within each specimen. Double immunostaining of serial sections for HDGF, PCNA, and $\alpha$-smooth muscle actin with confocal imaging is shown in Figure 12. As shown by the representative cell in Figure 12a, the $\alpha$-smooth muscle actin positive cell (green cytoplasm) has an HDGF-positive nucleus (red). Similarly, as shown in Figure 12b, SMCs in the vascular wall that have an HDGF-positive nucleus are also PCNA positive (yellow with overlay of red and green).

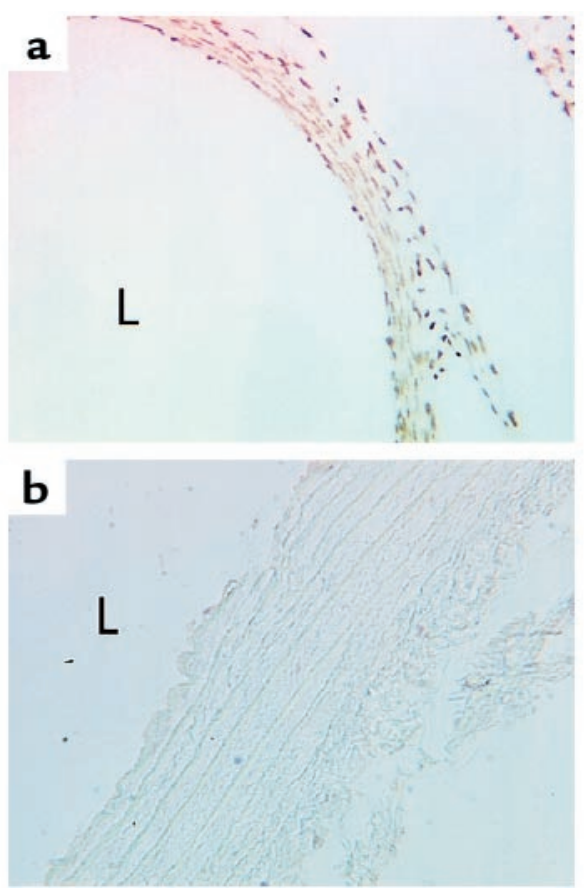

\section{Figure 9}

HDGF expression in fetal and adult rat aorta. Nineteen-day fetal (a) and adult rat thoracic aorta (b) sections were immunostained for $\operatorname{HDGF}(1: 1,000)$. HDGF-positive nuclei are brown with every nucleus positive in the fetal aorta (a), whereas only an occasional nucleus is seen to weakly stain in the adult aorta (b). $\mathrm{L}=$ lumen. 

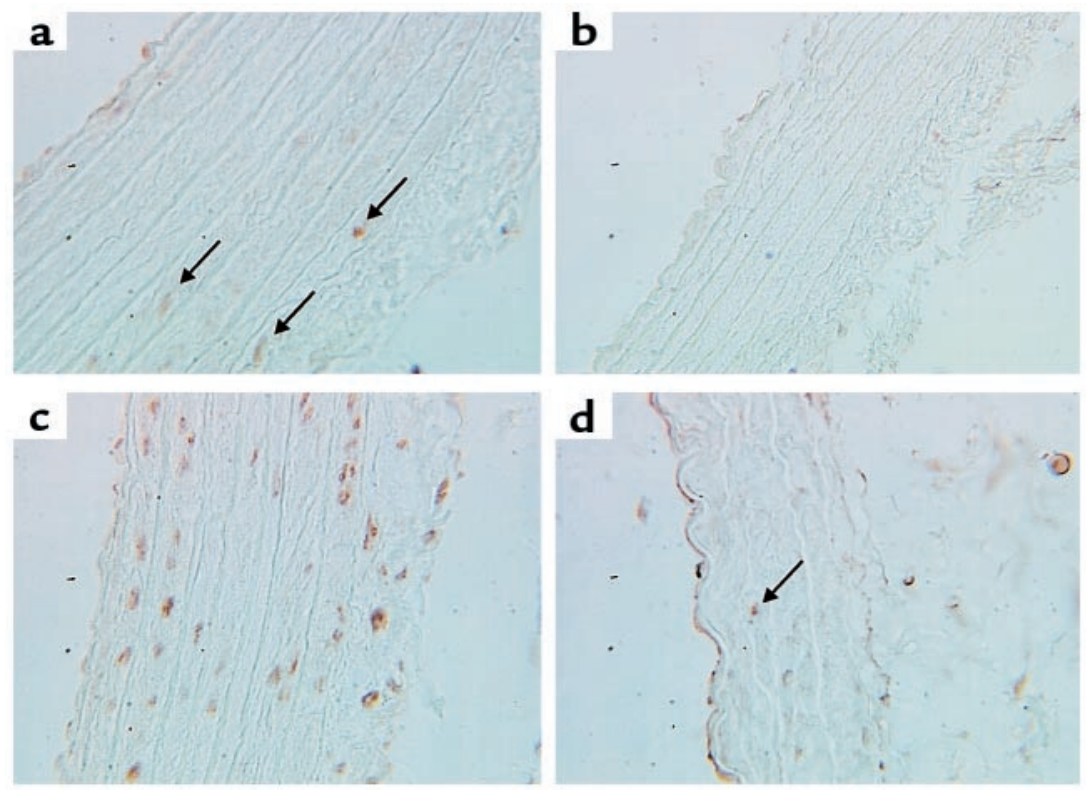

\section{Figure 10}

HDGF expression is increased in SMCs of the aorta after aortic constriction. HDGF immunostaining is shown in sham thoracic (a, arrows) and abdominal aorta (b) and in the thoracic aorta above the constriction (c, brown nuclei) and in the abdominal aorta below the constriction (d, arrow).

Therefore, proliferating SMC in atherosclerotic human carotid arteries also express HDGF.

\section{Discussion}

Identification of the factors regulating SMC growth is key to understanding vascular development and pathology. HDGF is a recently identified heparin-binding growth factor that is expressed in vascular SMCs during development and in response to injury, implicating it as a regulator of vascular SMC growth. The specific role of HDGF in vascular development and lesion formation is unknown. Our data and that of others suggest that it may have dual roles both as a secreted growth factor that acts in an autocrine or paracrine manner and as a nuclear factor potentially regulating gene expression or DNA synthesis.

Results of the present study demonstrated that HDGF mRNA is expressed in cultured rat aortic SMC, suggesting that this factor may regulate vascular development and disease via an intracrine, autocrine, or paracrine mechanism. HDGF mRNA expression in SMCs is increased in response to serum stimulation, suggesting a link to cellular proliferation. Indeed, exogenous HDGF was directly mitogenic for rat aortic SMCs in culture. HDGF-induced mitogenesis is not specific for SMCs, as HDGF purified from the conditioned media of HuH-7 cells and Cos-7 cells transfected with an HDGF-expression plasmid stimulated proliferation in Swiss 3T3 fibroblasts and HuH-7 cells (13). HDGF has also been purified from a cell line of rat metanephrogenic mesenchymal cells (7.1.1 cells) using an endothelial mitogenic assay. Thus, like other growth factors (FGF, PDGF, IGF-1), HDGF appears to be mitogenic for many cell types.
Although HDGF is a secreted protein $(13,14)$ previously thought to be exclusively cytoplasmic, and is an exogenous mitogen for SMCs, HDGF is detected in the nucleus of SMCs in the rat fetal aorta, in the rat adult aorta proximal to banding, and in neointimal cells of the injured rat carotid artery. Additionally, overexpressed recombinant epitope-tagged HA-HDGF and GFP-HDGF localized to the nucleus of cultured SMCs, replicating their DNA, and endogenous HDGF was detected in cultured SMC nuclear extracts. These data are the first to demonstrate that HDGF is also a nuclear protein. The differences in HDGF localization observed in our studies (nuclear) compared with those in the study of Oliver and Al-Awqati demonstrating that HDGF is localized in the cytoplasm of rat metanephrogenic mesenchymal cells (14) could be secondary to the use of different antibodies. Oliver and AlAwqat demonstrated cytoplasmic localization of HDGF using antibodies against the $\mathrm{NH}_{2}$-terminus of the protein. HDGF COOH-terminal cleavage products of $14-24 \mathrm{kDa}$ have been described from $\mathrm{HuH}-7$ cell-conditioned media (13) and 7.1.1 cell-conditioned media (14), supporting post-translational processing of HDGF. Our results demonstrating nuclear localization of HDGF in tissues was derived with an antibody directed against the $\mathrm{COOH}$-terminus. It is possible that the HDGF protein is modified after translation or cellular uptake such that only a portion of the protein, including the $\mathrm{NH}_{2}$-terminus, is retained in the cytoplasm. However, our data demonstrating that HAHDGF is exclusively expressed in the nucleus of proliferating SMC in culture were generated using an anti-HA antibody that targets the HA protein fused to the $\mathrm{NH}_{2}$-terminus of HDGF. Thus, it appears that the 

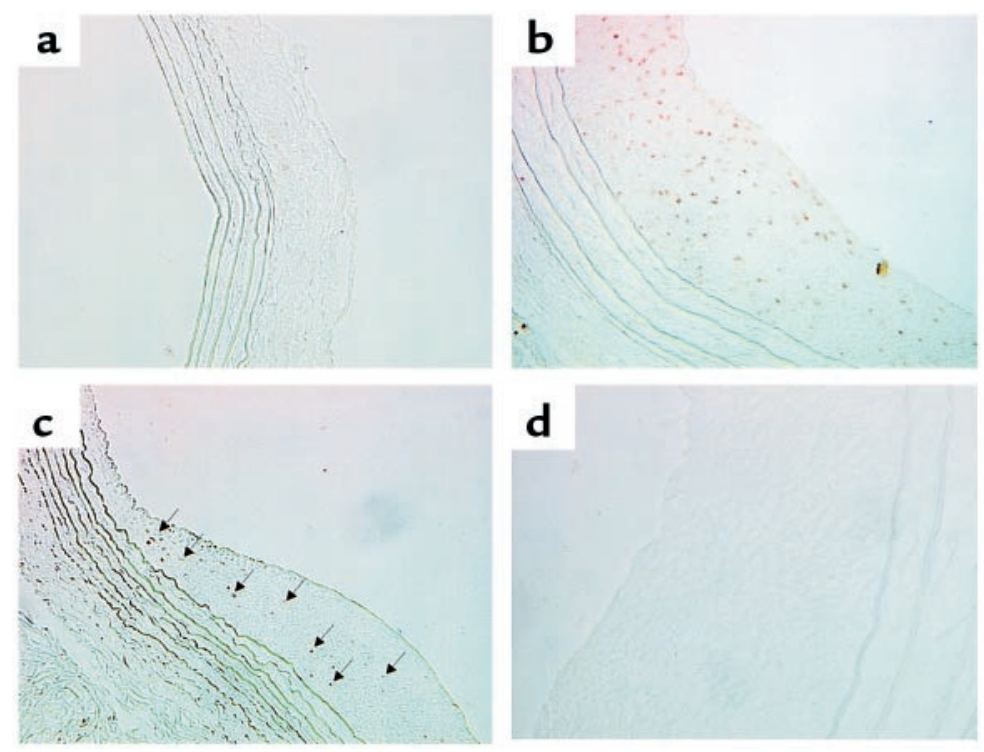

\section{Figure 11}

HDGF expression in the neointima after rat carotid artery balloon injury. HDGF immunostaining in sham (a) rat carotid arteries and at 14 days (b) and 28 days (c) after balloon injury. Brown nuclei (b and arrows in c) demonstrate positive HDGF immunostaining. $\mathbf{d}$ is a control serial section of $\mathbf{b}$ lacking the HDGF primary antibody.

mitogenic. More likely, HDGF has dual functions including acting as an exogenous growth factor to exert its effect and as a nuclear protein.

Immunohistochemistry demonstrated that HDGF is expressed in the nucleus of SMCs of the vessel wall in response to several different models of vascular injury as well as during development, suggesting that HDGF expression is a conserved response to vascular injury. Other investi-

full-length protein is expressed exclusively in the nucleus of proliferating SMCs.

A more likely explanation for the differential localization of HDGF is that HDGF resides in both the cytoplasm and nucleus depending on the cell type, the phase of the cell cycle, and its specific function. Previous studies demonstrating cytoplasmic localization of HDGF did not map expression of HDGF in relation to the proliferative state (14). Interestingly, HDGF shares the highest sequence homology with the high-mobility group-1 (HMG-1) protein (12). HMG-1 is a DNAbinding protein involved in DNA replication and expressed in both the cytoplasm and the nucleus depending on the growth and differentiation state and the specific cell type $(20,21)$. Additionally, HDGF contains a bipartite nuclear localization sequence that is conserved in other HDGF-related genes recently cloned from testis (17). The ability of HDGF to bind heparin, a characteristic of DNA binding proteins, further suggests a nuclear role for HDGF.

The specific function of HDGF in the nucleus and the mechanisms whereby exogenous HDGF stimulates cellular growth are unknown. It is unclear whether exogenous HDGF and nuclear HDGF stimulate SMC proliferation via distinct mechanisms or whether exogenous HDGF is taken up by receptor internalization and translocates to the nucleus to exert its effect on cellular growth. Nuclear localization of other exogenous heparin-binding growth factors such as FGF (22) has been demonstrated, and this may represent a conserved mechanism for targeting specific cellular effects. Results of the present study demonstrating that transfection of HA-HDGF results in nuclear localization of the resultant protein in scattered isolated cells, and not in cells in close proximity to the positive cells, suggests that the expressed protein directly translocates to the nucleus and is not locally secreted, taken up, and subsequently translocated to the nucleus. However, this does not account for the fact that exogenous HDGF is gators have shown that in response to injury, SMCs produce FGF and PDGF, both potent SMC mitogens. It is unclear whether HDGF expression is triggered by vascular injury directly or whether HDGF lies downstream of earlier signaling events. The 8- to 24-hour lag in the increase in HDGF mRNA in response to serum stimulation suggests that HDGF is not an early growth response gene but lies further downstream as a secondary regulator of cell growth. HDGF expression in SMCs does not appear to lie downstream of thrombin or PDGF, as their treatment did not alter HDGF mRNA levels. It is also unclear at this time whether
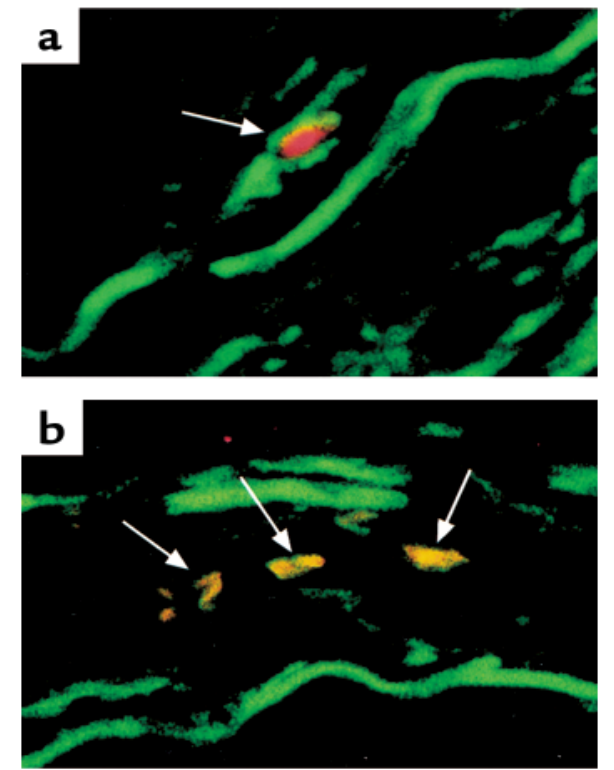

\section{Figure 12}

HDGF expression in SMCs of atherosclerotic human carotid arteries. Representative sections from a human carotid surgical endarterectomy specimen were double immunostained for (a) HDGF (red) and $\alpha$-smooth muscle actin (green) and (b) PCNA (green) and HDGF (red) to produce a yellow nucleus when the images are overlaid. 
translocation of HDGF to the nucleus is necessary for the induction of DNA synthesis or whether nuclear HDGF is acting as a regulator of gene transcription. Identification of the specific receptor that mediates the mitogenic effect of exogenous HDGF, the molecular mechanisms whereby this signal results in SMC growth, and the role of HDGF in the nucleus may provide important insight into the molecular mechanisms that regulate SMC growth during development and in response to vascular injury.

\section{Acknowledgments}

This work was supported in part by University of Virginia Children's Medical Center (A.D. Everett) and the University of Virginia Cardiovascular Research Center (A.D. Everett), by NHLBI R01 HL62522 (C.A. McNamara), and by the High Blood Pressure Research Training Grant NIH HL07355 (M.E. Matsumura). The authors thank I. Kron, University of Virginia Department of Thoracic Cardiovascular Surgery for providing human carotid artery specimens, and T. Stoops for excellent technical assistance.

1. Yoshida, Y., Mitsumata, M., Yamane, T., Tomikawa, M., and Nishida, K. 1988. Morphology and increased growth rate of atherosclerotic intimal smooth-muscle cells. Arch. Pathol. Lab. Med. 112:987-996.

2. Langlands, K., Yin, X., Anand, G., and Prochownik, E.V. 1997. Differential interactions of id proteins with basic-helix-loop-helix transcription factors. J. Biol. Chem. 272:19785-19793.

3. Clowes, A.W., Reidy, M.A., and Clowes, M.M. 1983. Kinetics of cellular proliferation after arterial injury. I. Smooth muscle growth in the absence of endothelium. Lab. Invest. 49:327-333.

4. Raines, E.W., and Ross, R. 1993. Smooth muscle cells and the pathogenesis of the lesions of atherosclerosis. Br. Heart J. 69(Suppl.):S30-S37.

5. McNamara, C.A., et al. 1993. Thrombin stimulates proliferation of cultured rat aortic smooth muscle cells by a proteolytically activated receptor. J. Clin. Invest. 91:94-98.

6. Winkles, J.A., et al. 1987. Human vascular smooth muscle cells both express and respond to heparin-binding growth factor I (endothelial cell growth factor). Proc. Natl. Acad. Sci. USA. 84:7124-7128.

7. Ross, R., Raines, E.W., and Bowen-Pope, D.F. 1986. The biology of platelet-derived growth factor. Cell. 46:155-169.

8. Fingerle, J., and Kraft, T. 1987. The induction of smooth muscle cell proliferation in vitro using an organ culture system. Int. Angiol. 6:65-72.

9. Guyton, J.R., and Karnovsky, M.J. 1979. Smooth muscle cell proliferation in the occluded rat carotid artery: lack of requirement for luminal platelets. Am. J. Pathol. 94:585-602.

10. Wilcox, J.N., Smith, K.M., Williams, L.T., Schwartz, S.M., and Gordon, D. 1988. Platelet-derived growth factor mRNA detection in human atherosclerotic plaques by in situ hybridization. J. Clin. Invest. 82:1134-1143.

11. Vlodavsky, I., et al. 1987. Endothelial cell-derived basic fibroblast growth factor: synthesis and deposition into subendothelial extracellular matrix. Proc. Natl. Acad. Sci. USA. 84:2292-2296.

12. Nakamura, H., et al. 1994. Molecular cloning of complementary DNA for a novel human hepatoma-derived growth factor. Its homology with high mobility group-1 protein. J. Biol. Chem. 269:25143-25149.

13. Nakamura, H., et al. 1989. Partial purification and characterization of human hepatoma-derived growth factor. Clin. Chim. Acta. 183:273-284.

14. Oliver, J.A., and Al-Awqati, Q. 1998. An endothelial growth factor involved in rat renal development. J. Clin. Invest. 102:1208-1219.

15. Heller, F.A., Xue, C., and Fisher, A.E.A.D. 1998. Expression and mapping of protein phosphate $2 \alpha$ in the developing rat heart. Pediatr. Res. 43:68-76.

16. Dignam, J.D., Lebovitz, R.M., and Roeder, R.G. 1983. Accurate transcription initiation by RNA polymerase II in a soluble extract from isolated mammalian nuclei. Nucleic Acids Res. 11:1475-1489.

17. Izumoto, Y., Kuroda, T., Harada, H., Kishimoto, T., and Nakamura, H. 1997. Hepatoma-derived growth factor belongs to a gene family in mice showing significant homology in the amino terminus. Biochem. Biophys. Res. Commun. 238:26-32.

18. Clowes, A.W., Clowes, M.M., and Reidy, M.A. 1986. Kinetics of cellular proliferation after arterial injury. III. Endothelial and smooth muscle growth in chronically denuded vessels. Lab. Invest. 54:295-303.

19. Everett, A.D., Tufro-McReddie, A., Fisher, A., and Gomez, R.A. 1994. Angiotensin receptor regulates cardiac hypertrophy and transforming growth factor-beta 1 expression. Hypertension. 23:587-592.

20. Mosevitsky, M.I., Novitskaya, V.A., Iogannsen, M.G., and Zabezhinsky, M.A. 1989. Tissue specificity of nucleo-cytoplasmic distribution of HMG1 and HMG2 proteins and their probable functions. Eur. J. Biochem. 185:303-310.

21. Bonne-Andrea, C., Harper, F., Puvion, E., Delpech, M., and De, R. 1986. Nuclear accumulation of HMG1 protein is correlated to DNA synthesis. Biol. Cell. 58:185-194.

22. Lindner, V., and Reidy, M.A. 1993. Expression of basic fibroblast growth factor and its receptor by smooth muscle cells and endothelium in injured rat arteries. An en face study. Circ. Res. 73:589-595. 